\title{
Molecular characterization of gastric-type endocervical adenocarcinoma using next-generation sequencing
}

\author{
Swati Garg ${ }^{1} \cdot$ Teddy S. Nagaria ${ }^{2} \cdot$ Blaise Clarke ${ }^{2,3} \cdot$ Orit Freedman $^{4} \cdot$ Zanobia Khan $^{2,3} \cdot$ Joerg Schwock $^{2,3}$. \\ Marcus Q. Bernardini ${ }^{3} \cdot$ Amit M. Oza ${ }^{3} \cdot$ Kathy $\operatorname{Han}^{3} \cdot$ Adam C. Smith $\mathbb{1}^{2,5} \cdot$ Tracy L. Stockley ${ }^{1,2,5}$. \\ Marjan Rouzbahman ${ }^{2,3}$
}

Received: 13 November 2018 / Revised: 16 May 2019 / Accepted: 17 May 2019 / Published online: 15 July 2019

(c) The Author(s), under exclusive licence to United States \& Canadian Academy of Pathology 2019

\begin{abstract}
Gastric-type endocervical adenocarcinoma is an uncommon aggressive type of endocervical adenocarcinoma that is not associated with human papillomavirus (HPV). At present, this tumor is classified under the spectrum of mucinous carcinoma of the uterine cervix. The clinical stage of gastric-type endocervical adenocarcinoma at the time of diagnosis is usually more advanced compared to the HPV-associated endocervical adenocarcinoma. Widespread dissemination to unusual sites, such as omentum, peritoneum, and distant organs, can be present. Owing to its rare incidence, diagnostic dilemmas, and aggressive behavior, clinical management can be challenging. In this study, we aimed to elucidate the molecular characteristics of these tumors by using next-generation sequencing (NGS) to assess 161 unique cancer-driver genes for single-nucleotide and copy-number variations, gene fusions, and insertions/deletions within gastric-type endocervical adenocarcinoma tumors. In total, 92 variants were detected across the 14 samples tested ( 7 variants on average per tumor). TP53 was the most recurrently mutated gene followed by MSH6, CDKN2A/B, POLE, SLX4, ARID1A, STK11, BRCA2, and MSH2. Abnormal p53 expression was observed in nine cases by immunohistochemistry, of which TP53 variants were present in four cases. MDM2 gene amplification in 12q15 (69202190-69233452) locus was seen in two cases that express normal p53 levels by immunohistochemistry. Four cases had STK11 null (frameshift/nonsense) variants, three of which were previously reported in Peutz-Jeghers syndrome. Overall, genes that are implicated in DNA damage, repair, cell cycle, Fanconi anemia pathway, and the PI3K-AKT signaling pathways were found to be mutated. Of note, genes known to have acquired and/or inherited variants in endometrial tumors were enriched within our cohort. In conclusion, our study shows the genetic heterogeneity of gastric-type endocervical adenocarcinoma with some potentially actionable molecular alterations, which highlights the importance of further molecular characterization for better identification of this rare entity, and hence better clinical management.
\end{abstract}

These authors contributed equally: Swati Garg, Teddy S. Nagaria

Marjan Rouzbahman

marjan.rouzbahman@uhn.ca

1 Advanced Molecular Diagnostics Laboratory, Princess Margaret Cancer Centre, University Health Network, Toronto, ON, Canada

2 Department of Laboratory Medicine and Pathobiology, University of Toronto, Toronto, ON, Canada

3 Princess Margaret Cancer Centre, University Health Network, Toronto, ON, Canada

4 Lakeridge Health Centre, Oshawa, ON, Canada

5 Genome Diagnostics, Department of Clinical Laboratory Genetics, Laboratory Medicine Program, University Health Network, Toronto, ON, Canada

\section{Introduction}

The majority of endocervical adenocarcinomas are associated with human papillomavirus (HPV) mainly HPV types 16 and 18. The non-HPV-related tumors are a heterogenous group that are much less frequent and often pose diagnostic and management challenges. Within the nonHPV-related group, there is a relatively recently recognized entity known as gastric-type endocervical adenocarcinoma [1-4]. Japanese research groups first described this uncommon entity in the 1990s coining the term "gastrictype" for its resemblance to the gastric and pancreatobiliary epithelial lining [5-8]. Over the past two decades, characterization of this entity has defined a spectrum of endocervical tumors that range from minimal deviation 
adenocarcinoma of mucinous type, also termed as adenoma malignum-a well-differentiated variant of gastric-type endocervical adenocarcinoma-to the poorly differentiated gastric-type endocervical adenocarcinoma $[9,10]$. The most recent World Health Organization (WHO) classification of Tumors of Female Reproductive Organs classifies gastrictype endocervical adenocarcinoma as a distinct type of adenocarcinoma under the category "mucinous carcinoma" of the uterine cervix, establishing this type of tumor as a distinct entity with specific histological features, immunohistochemistry profile, and clinical behavior unique from the usual type endocervical adenocarcinoma [11, 12]. Gastric-type endocervical adenocarcinoma is characterized by tumor cells showing voluminous clear or pale eosinophilic cytoplasm, distinct cell borders, and at least moderate nuclear atypia. These tumors may contain areas that are indistinguishable from minimal deviation adenocarcinoma. The neoplastic cells contain acidic mucin similar to minimal deviation adenocarcinoma and express immunomarkers similar to gastric mucus cells, with the expression of HIK1083, lysozyme, and pepsinogen II [13, 14]. Overall, there is limited data on immune-phenotypic characterization of non-HPV-associated endocervical glandular lesions and gastric-type endocervical adenocarcinoma [12]. The immunoprofile of gastric-type endocervical adenocarcinoma has been explored in few recent studies [13, 14]; however, currently there is no ancillary study that is diagnostic of this entity and therefore the diagnosis is made based on the classic histological features, excluding other tumors in the differential diagnosis and negative HPV status.

It has been documented that gastric-type endocervical adenocarcinoma behaves more aggressively than HPVassociated endocervical adenocarcinoma and often displays a more widespread involvement at the time of presentation $[15,16]$.

In the current work, we aim to explore the genomic landscape of this rare yet aggressive tumor type to identify molecular features that may lead to a better understanding of the pathogenesis of this rare entity, in hopes of providing new diagnostic or therapeutic modalities to enhance clinical management.

\section{Methods}

After obtaining institutional authorization and Research Ethics Board approval, we identified patients with a diagnosis of gastric-type endocervical adenocarcinoma at University Health Network between 2008 and 2016. Fourteen patients had in-house pathology material available for study. Clinical parameters obtained from the electronic patient record included: age, clinical presentation, stage at presentation, type of surgery, adjuvant radiation or chemotherapy, follow-up, recurrence, last follow-up, and survival status at the time of last follow-up. The pathology gross examinations as well as available radiology reports were reviewed to document the endocervical location of the tumors. All available hematoxylin-eosin (H\&E)-stained slides were re-assessed by two gynecologic pathologists (MR, BC) and the diagnosis was confirmed using the histologic criteria as originally described by Kojima et al. with tumor cells demonstrating voluminous clear or pale eosinophilic cytoplasm, distinct cell borders, and moderate-tomarked nuclear atypia. All cases were tested for HPV using linear array analysis to confirm negative HPV status. The size and location of tumor, pathologic stage, presence of lymphovascular space invasion, and any additional pathology findings were documented.

\section{Immunohistochemistry}

Using standard methods, immunohistochemical staining for p53 (Leica NCL, clone: D07, dilution: 1/1000), p16 (BD Pharmingen 550834 TRIS-EDTA pH 9.0, dilution 1/400 with $2 \mathrm{~h}$ incubation), PAX8 (Biocare AC1438 TRIS-EDTA $\mathrm{pH}$ 9.0, dilution $1 / 200$ with overnight incubation), Mismatch Repair markers: MSH2 (Pharmingen G219-1129 low temperature TRIS-EDTA pH 9.0 dilution $1 / 500$ with $1 \mathrm{~h}$ incubation), MSH6 (Transduction Lab 44/MSH6 TRISEDTA pH 9.0, dilution 1/300 with overnight incubation), MLH1 (Dako ES05 low temperature TRIS-EDTA pH 9.0, prediluted with $1 \mathrm{~h}$ incubation), and PMS2 (Pharmingen A16-4 TRIS-EDTA pH 9.0, dilution 1/200 with $1 \mathrm{~h}$ incubation) was performed.

Immunohistochemical staining was carried out using the standard streptavidin-biotin complex technique after microwave antigen retrieval on 4-micron sections of formalin-fixed, paraffin wax-embedded tissue. Appropriate positive and negative controls were run in parallel. The immunostained slides were reviewed and scored by two pathologists (MR and $\mathrm{BC}$ ).

Interpretation of p16 immunostaining was reported as negative, focal, or diffuse. p53 was reported as normal expression (wild type) or abnormal expression (overexpressed or null type). HER2 immunostained slides were scored using the College of American Pathologists guidelines for breast carcinomas [17].

\section{Fluorescence in situ hybridization (FISH)}

Amplification of Her-2/neu was evaluated using the PathVysion DNA Probe Kit (Abbott-Vysis, Downers Grove, IL), which uses a dual-color probe for determining the number of copies of both Her-2/neu (orange) and the chromosome 17 centromeres (green). The kit was used following the manufacturer's instructions. Slides containing 
4- $\mu$-thick paraffin-embedded tissue sections of studied cervical tumor cases and Her-2/neu normal and cut-off breast control slides (Abbott-Vysis probechek pathvysion, Downers Grove, IL) were used for FISH and were stored at $-20{ }^{\circ} \mathrm{C}$ until ready to score.

Analysis was done using a fluorescence microscope (Zeiss Axio Imager, Gottingen, Germany) equipped with a triple bandpass filter set (DAPI/Green/Orange), dual bandpass filter set (Green/Orange), and single bandpass filters (DAPI, Spectrum Orange, Spectrum FITC). Image capture was done using a digital ProgRes MF video camera (Jenoptik, Germany) and the fluorescence image acquisition software ISIS (MetaSystems, Germany). Slide quality was assessed following the updated 2013 recommendations from the ASCO/CAP Recommendations for HER2 Testing in Breast Cancer [18]. Positive and negative control slides for each FISH run was assessed and scored to determine acceptability of FISH run. At least 30 nonoverlapping tumor nuclei were scored using pathologistcircled $\mathrm{H} \& \mathrm{E}$ as a guide. The ratio of HER2/neu signals (orange) to chromosome 17 centromere signals (green) along with the average counts per signal was determined with ratios of $<2.0$ considered non-amplified and those $\geq 2.0$ amplified. Additional nuclei were scored for challenging and equivocal results as recommended within the ASCO/CAP guidelines [18].

\section{Molecular profiling and variant analysis}

Molecular testing using formalin-fixed paraffin-embedded tumor specimens was performed in the Advanced Molecular Diagnostics Laboratory at Princess Margaret Cancer Center (Toronto, Canada). DNA and RNA were co-isolated from 14 gastric type endocervical carcinomasusing Maxwell(R) RSC RNA formalin-fixed paraffin-embedded Kit (Promega, Madison, WI). Next-generation sequencing (NGS) was performed using the Oncomine Comprehensive Assay v3 (Thermo Fisher, Waltham, MA), which assesses singlenucleotide variations, copy-number variation gene fusions, and insertions/deletions from 161 cancer-driver genes. For each sample, $20 \mathrm{ng}$ of DNA and $40 \mathrm{ng}$ RNA were used to prepare sequencing libraries on an automated library preparation station (Ion Chef System, Thermo Fisher, Waltham, MA). Libraries were sequenced using the 540 chip on the S5XL platform (Thermo Fisher, Waltham, MA). Postsequencing analysis was performed using the Ion Reporter Software (Thermo Fisher, Waltham, MA) and the Oncomine Knowledgebase Reporter (Thermo Fisher, Waltham, MA), with default filters for copy-number variations and fusions, and with slight modifications for single-nucleotide variations and insertions/deletions to retain only variants with an allele frequency $\geq 5 \%$ and coverage $\geq 50 \times$. Common single-nucleotide variations (as assessed by presence in University of California, Santacruz genome browser (UCSC) [19] and synonymous changes were excluded from analysis using Oncomine Knowledgbase Reporter. Additional manual variant review removed variants if they were reported as benign/likely benign in the clinvar [20] database or if present in any of the following reference population variant databases with an minor allele frequency of $>1 \%$ : 1000 Genomes (Phase1 release v3.20101123, 1000 Genomes Phase 3 release v5.20130502 [21]; ESP6500SI-V2 dataset of the exome sequencing project [22] annotated with seattleseqannotation137; Exome Aggregation Consortium [exac] release 0.3 [23]; and genome build GRCh37.p13).

\section{Results}

\section{Patient characteristics}

Mean age at diagnosis was 56 years (range; 35-79). The most common clinical presentation was vaginal bleeding/ discharge (10 cases). Seven of 14 cases had a Pap smear reported as abnormal. The diagnoses made on Pap smear were "abnormal glandular cells" in four and "adenocarcinoma" in three of the seven cases. Ethnicity breakdown was as follows: Asian background (6/14 including 2 Chinese, 1 Philippino, and 3 South Asian: Pakistan/Bangladesh/India), Caucasian (7/14), and African Canadian (1/14).

The clinical stage at presentation was: IA2 (1/14), IB1 (6/16), IIA (1/14), IIB (2/14), IIIB (3/14), and IVB (1/14). Clinical follow-up information was available in 14 cases. The mean follow-up period was 40 months (range $10-103$ months). Five of $14(35 \%)$ died of disease and nine of $14(65 \%)$ were alive at the time of last follow-up. Four patients (patients 1, 5, 12, and 13 in Table 1) were alive with disease and the rest were alive without disease at the time of collecting the data. Our data showed that 12 patients had total abdominal hysterectomy and bilateral salpingectomy and/or oophorectomy (with lymph node dissection in five), and two patients had unresectable disease at the time of diagnosis. Eleven patients had adjuvant chemotherapy and radiation following surgery. Five of the 14 patients were shown to have lymph node involvement, and lymphovascular invasion was present in 9 patients of 14 cases $(64 \%)$. Family history of cancer was noted in 7 patients. Family history breakdown included colon cancer (5/14), pancreatic cancer (1/14), breast cancer (1/14), and ovarian cancer (1/14). One individual had family history of both breast and colon cancers. None of our cases had clinical manifestations or familial history of Peutz-Jeghers syndrome. A summary of patients' characteristics and molecular findings is provided in Table 1. 


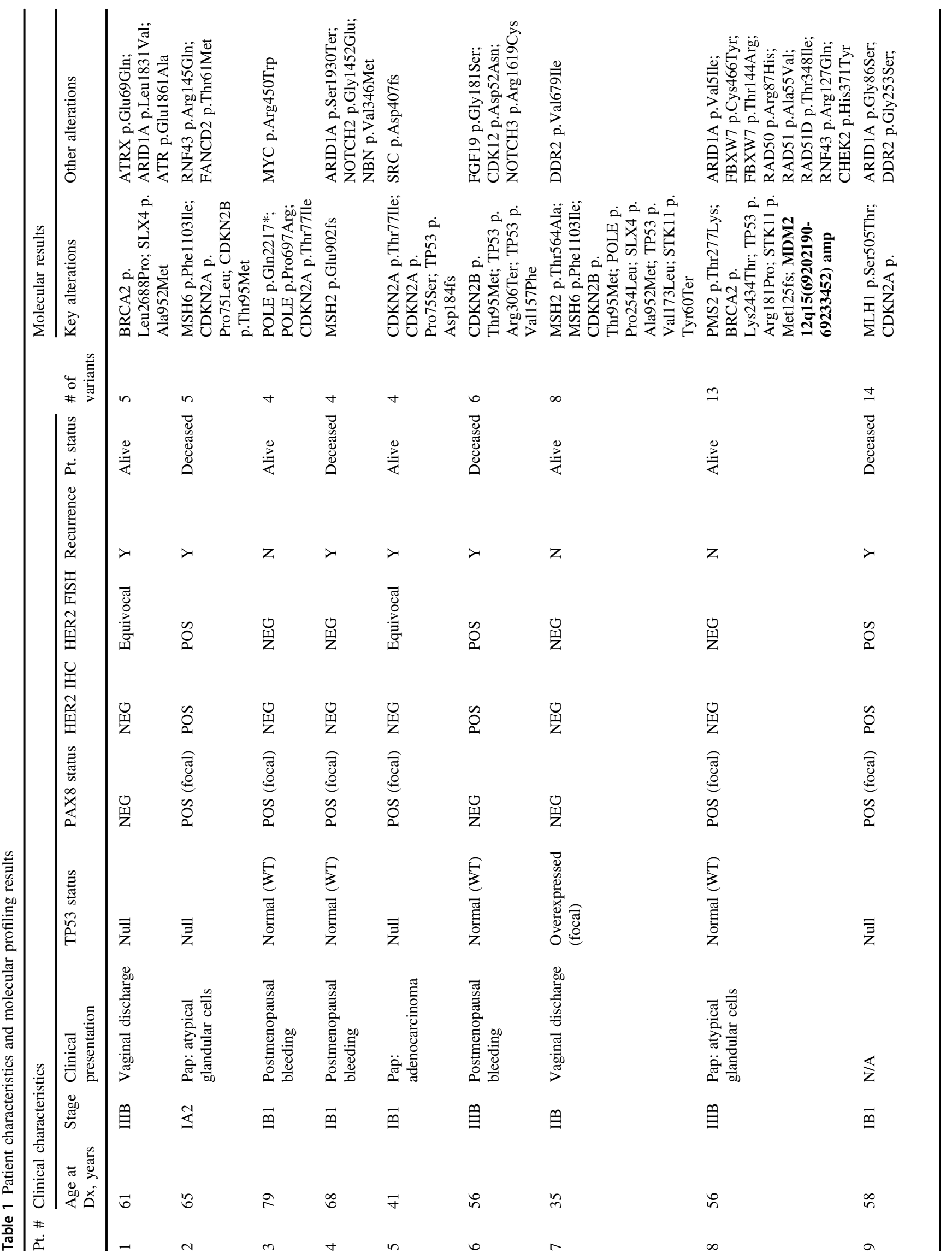




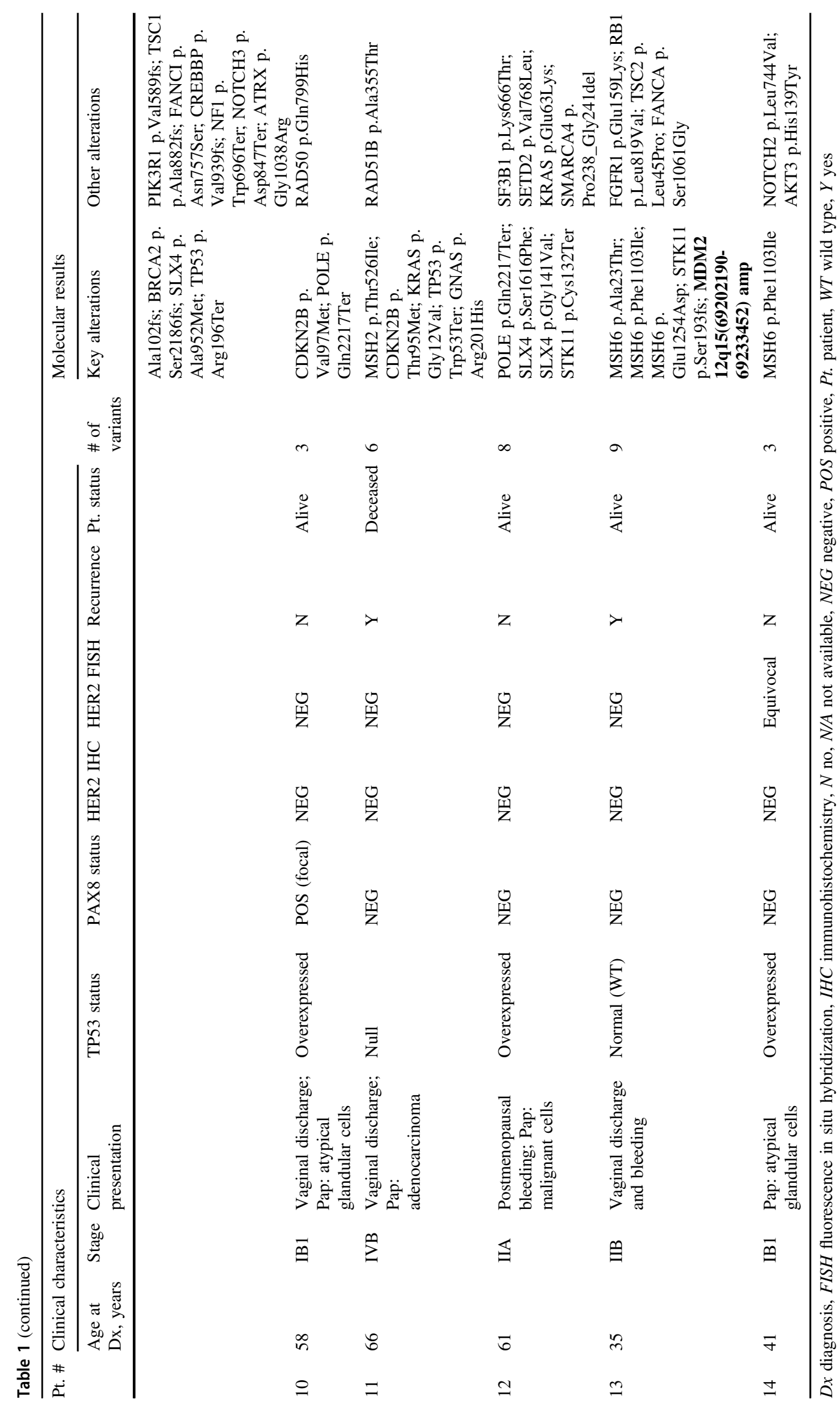




\section{HER2 amplification and immunohistochemical staining of p53, p16, PAX8 in gastric type endocervical adenocarcinoma}

Immunohistochemical analysis of these cases showed that 12 cases were negative for $\mathrm{p} 16,1$ showed focal staining, and 1 was patchy positive. PAX8 was focal weak positive in 7 cases and negative in 5 cases (not performed on 2 cases). Abnormal p53 expression was seen in 9 cases ( 3 cases were diffuse overexpression, 6 cases were null expression), whereas 5 cases showed a wild-type p53 pattern. There was no correlation observed between p53, p16, and PAX8 expression patterns and patient status.

Immunohistochemistry for Mismatch Repair markers was performed. All cases showed intact expression, except for one that showed loss of MLH1/PMS2. Subsequent PCR revealed $M L H$ promoter methylation in this particular case.

FISH results revealed that 2 of the 12 (20\%) patients exhibited HER 2 amplification by FISH; 3 showed equivocal results and 7 were negative for HER2 amplification by FISH. Both patients with HER2 amplification had died of disease at the time of last follow-up. By immunohistochemistry, there was positive staining for HER2 in the same two cases that showed amplification on FISH $(100 \%$ correlation with FISH results).

\section{Molecular findings}

A total of 92 variants were detected across 14 gastric-type endocervical adenocarcinoma patient tumor samples following NGS variant filtration as described (Table 1). Variants were identified most frequently in the TP53, MSH6, CDKN2A/B, POLE, SLX4, ARID1A, STK11, BRCA2, and $M S H 2$ genes (Fig. 2A). Genes that are associated with one or many of the following pathways are found to be mutated in our dataset as follows, homologous recombination deficiency (BRCA2, NBN, RAD50, RAD51, RAD51B) mismatch repair (MLH1, MSH2, MSH6, and PMS2), cell cycle (TP53, MDM2, CREBBP, RB1, MDM2, CDKN2A/B, ATR, MYC, CHEK2), Fanconi anemia pathway (FANCA, FANCD2, FANCI, SLX4, ATR), NOTCH signaling (NOTCH2 and NOTCH3), and/or PI3K-AKT signaling pathway (TSCl/2, AKT3, FGF19, STK11, MYC, FGFRI) as well as genes reported in endometrial cancers (POLE, ARIDIA, KRAS, $F B X W 7$ ). Most of the genes cluster together on common pathways and seem to belong to a common network (Fig. 2B).

Missense variants in the mismatch repair genes $(M L H 1$, MSH2, MSH6, and/or PMS2) were detected in 7 of the 14 gastric-type endocervical adenocarcinoma tumors, whereas in 1 case there was frameshift $M S H 2$ variant detected; however, all 8 cases exhibited intact proteins in immunohistochemistry.

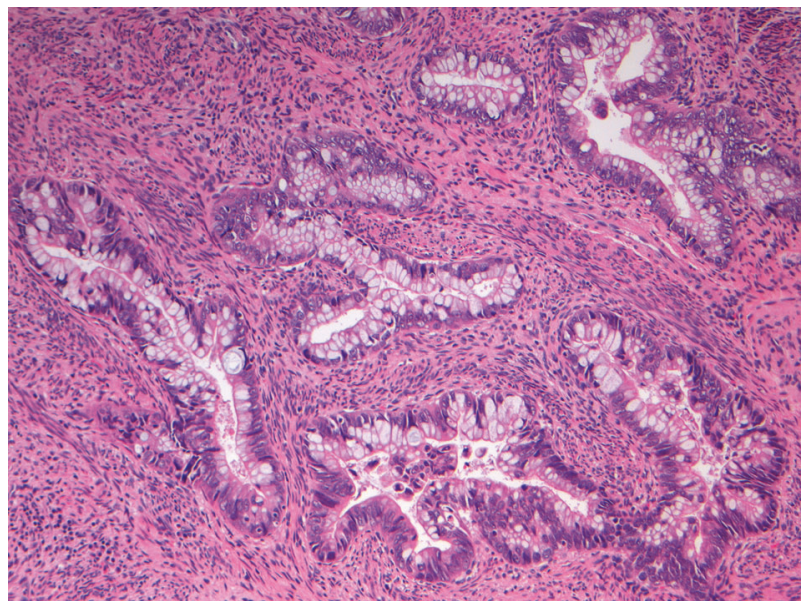

Fig. 1 Gastric-type endocervical adenocarcinoma. Hematoxylin and eosin staining showing malignant glands with distinct cell membrane, voluminous clear cytoplasm, and basally located nuclei with moderate nuclear atypia

Our data found that there were 4 cases with STK11 null (frameshift/nonsense) variants (Table 2). Three of the 4 variants were previously reported as pathogenic variants in human gene mutation database. Two of these patients had no family history of cancer. One had history of colon cancer in her mother and another one had history of colon cancer in her sister.

There were distinct mutational patterns observed in patients differing in their p53 immunohistochemical status. Interestingly, two of the five cases who had normal p53 levels by immunohistochemistry showed MDM2 gene amplification in 12q15 (69202190-69233452) locus. Three of the four tumors with overexpressed p53 exhibited $P O L E$ variants. $C D K N 2 A / B$ variants were predominant in tumors exhibiting p53 expression loss. There was no correlation observed between POLE mutation status and overall number of mutations, although the panel size may not be sufficiently large to detect correlations. We reviewed all material on tumors with POLE mutation again to exclude the possibility of endometrial adenocarcinomas extending to the cervix. Upon review of tumors with POLE variants, we confirmed the presence of main tumor masses grossly centered in the cervix/upper endocervical canal. Histological review of those cases by two pathologists demonstrated classic histological features of gastric-type endocervical adenocarcinoma. Tumors with either normal or null TP53 expression were highly mutated and were enriched in genes found to be mutated in endometrial cancers, DNA damage, or repair pathways; whereas p53 overexpressed cells had no particular pathway enriched. KRAS, GNAS, and STK11 variants are described to be present in mutually exclusive patterns in lobular endocervical glandular hyperplasia (LEGH) [24]. We observed two cases that had KRAS 

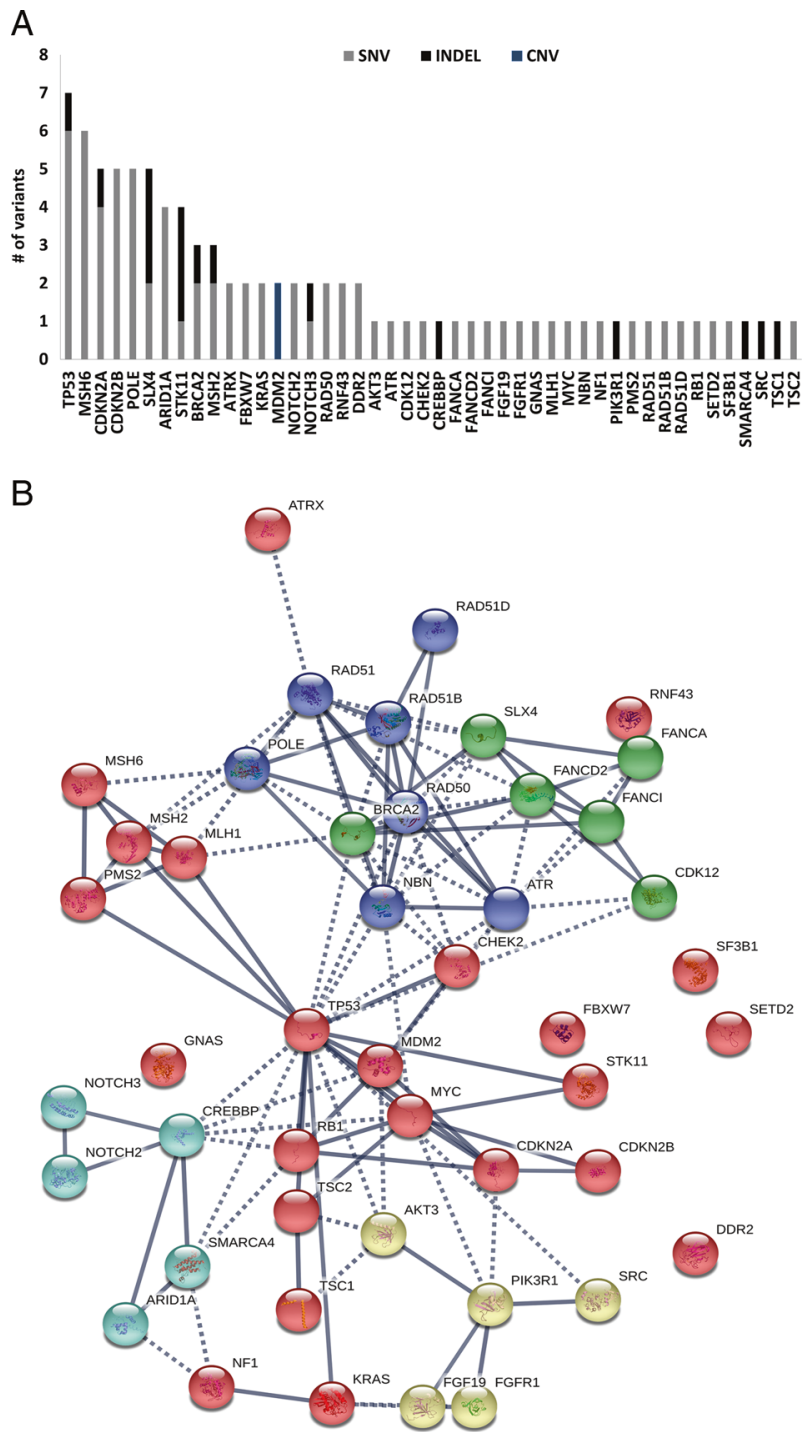

Fig. 2 Mutational profile of gastric type endocervical adenocarcinoma using Comprehensive Oncomine V3 assay $(n=14)$. A Overall distribution of variants across 14 samples. B Network analysis performed using STRINGS database among genes found to be mutated reveals that the mutated genes share common pathways. Network nodes (circles (O)) represent proteins and edges (lines/dashes (-/---)) represent the degree of connectivity. The thickness of the edges represents the degree of association between the two proteins

variants-one with KRAS p.Glu63Lys along with a pathogenic STK11 truncation variant (p.Cys132Ter). The other case had KRAS p.Gly12Val variant along with a GNAS hotspot p.Arg201His variant. Both tumors were negative for HPV and for p16 expression.

\section{Discussion}

In this study, we reviewed the clinicopathologic features and explored the genomic landscape of gastric-type
Table 2 STK11 frameshift/truncation variants detected in our dataset

\begin{tabular}{|c|c|c|}
\hline Sample Id & STK11 variants & Family history status \\
\hline 8 & c.369delG (p.Met125fs) & None \\
\hline 12 & c.396C $>$ A (p.Cys132Ter) & $\begin{array}{l}\text { Sister with } \\
\text { colon cancer }\end{array}$ \\
\hline 7 & c.180delC (p.Tyr60Ter) & $\begin{array}{l}\text { Mother with } \\
\text { colon cancer }\end{array}$ \\
\hline 13 & $\begin{array}{l}\text { c.577_581delTCCGA } \\
\text { (p.Ser193fs) }\end{array}$ & None \\
\hline
\end{tabular}

Variants in bold were found to be associated with Peutz-Jeghers syndrome

endocervical adenocarcinomas at our institution. Our clinical and demographic data are in concordant with previously published literature on this topic. Kojima et al. in their original study showed a significantly poorer 5-year prognosis of gastric-type endocervical adenocarcinoma in comparison to non-gastric-type endocervical adenocarcinoma [13]. Karamurzin et al. reported that disease-specific 5-year survival was $42 \%$ for gastric-type endocervical adenocarcinoma as opposed to $91 \%$ for HPV-related adenocarcinoma [16]. Contrary to the usual type of endocervical adenocarcinoma that typically recurs in a localized region in the pelvis, gastric-type endocervical adenocarcinoma disseminates more frequently to adnexa, omentum, liver, brain, and bone [12, 15, 16, 25]. In line with previous literature reports that showed gastric-type endocervical adenocarcinoma dissemination to unusual sites, our data showed peritoneal carcinomatosis in 3 patients and metastasis-associated pleural effusion and metastasis to mediastinal lymph nodes in 1 patient. Nine patients showed positive lymph-vascular invasion, along with five who showed lymph node involvement. This data supports that, owing to propensity for widespread dissemination, ovarianconserving surgery may not be a good therapeutic option for these patients $[12,16]$. Chemotherapy resistance to docetaxel and carboplatin-based regimen has also been reported, which poses a dilemma in managing gastric-type endocervical adenocarcinoma patients beyond surgical intervention $[16,26]$. Death is largely attributed to eventual disease recurrence, and this holds true even for the welldifferentiated gastric-type endocervical adenocarcinoma $[13,27]$.

Although the published data are limited regarding immunophenotype of gastric-type endocervical adenocarcinoma, Carleton et al. recently reported a detailed immunohistochemical profile of 45 cervical gastric-type adenocarcinomas [28]. They showed the utilization of immunostains such as CK7, CEA, CDX2, PAX8, MUC6, p53, ER, and PR to identify this challenging entity [14]. They reported positive staining with CK7 (47/47), MUC6 (17/21), carcinoembryonic antigen (25/31), carbonic 
anhydrase IX (20/24), PAX8 (32/47), CDX2 (24/47), CK20 (23/47), and p16 (18/47).

In our series, we identified positive CK7 (14/14), PAX8 (7/14), and p16 (2/14). In the 7 PAX8-positive cases of our series, the staining was focal and weak. Given overlapping features in histological characteristics and immunohistochemical profile between gastric-type endocervical adenocarcinoma and upper gastrointestinal tract and pancreatiobiliary adenocarcinomas, the distinction could be quite challenging in certain clinical settings. Although PAX8 staining is not present consistently in gastric-type endocervical adenocarcinoma, it is advisable that it be included in the panel of markers for differentiating these tumors from metastases as gastrointestinal adenocarcinomas are expected to be PAX8 negative [14]. In addition, p16 was negative in most of our patient tumors (12/14), one showed focal positive staining and another showed patchy staining pattern. The non-block-type (mosaic) staining of p16 has been previously reported in gastric-type endocervical adenocarcinoma. These results are in concordance with HPV-negative status of these tumors. Moreover, a diffuse-type $\mathrm{p} 16$ expression has also been reported in a rare subset of gastric-type endocervical adenocarcinoma despite negative molecular testing for HPV $[12,14,29]$. While the main differential diagnoses include other histotypes of cervical adenocarcinoma, endometrial adenocarcinomas, and metastases from outside the genital tract, still to date, no immunostains are known to be reliably specific in making such distinction. The immunomarker HIK1083 that highlights the specific type of mucin in these tumors is not commercially available in most countries. The diagnosis can be potentially challenging since the first manifestation of these mucinous adenocarcinomas can be with metastasis; therefore an advanced stage primary cervical adenocarcinoma should be distinguished from an advanced-stage gastrointestinal cancer $[8,12,24]$. Ancillary studies such as immunohistochemistry staining and molecular testing of HPV DNA in combination with macroscopic and microscopic findings should be used to assure proper identification of these tumors.

Emerging studies suggest that the spectrum of unusual non-HPV-associated endocervical lesions also includes benign and pre-malignant gastric-type glandular lesions [5$7,12,13,15,16,28,30-34]$. LEGH, a benign entity from the spectrum of gastric-type glandular lesions, has been reported to harbor genetic aberrations such as GNAS, KRAS, TP53, and STK11 variants [8, 24]. Co-existence of lesions consisting of both LEGH and gastric-type endocervical adenocarcinoma has also been reported [7, 12, 32, 35]. These mutational findings argue that a certain proportion of LEGH or atypical gastric-type glandular lesions are neoplastic rather than metaplastic, and may be directly associated with transformation and/or progression to gastric- type endocervical adenocarcinoma [12, 24]. It is still unclear at this point whether glandular lesions, such as LEGH, are a precursor to gastric-type endocervical adenocarcinoma. And, if indeed so, genetic aberrations that initiates or accelerates the progression toward malignant transformation remains to be further explored. In this series, however, we did not identify any associated LEGH.

Our molecular results support that gastric-type endocervical adenocarcinomas are quite heterogeneous in terms of genetic findings and exhibit alterations in several actionable genes, such as TP53, POLE, CDKN2A/B, the mismatch repair genes, Fanconi anemia genes, KRAS, $B R C A 2$, and ARID1A. Most of the altered genes cluster together on common pathways and seem to belong to a common network as follows: homologous recombination deficiency (BRCA2, NBN, RAD50, RAD51, RAD51B) mismatch repair (MLH1, MSH2, MSH6, and PMS2), cell cycle (TP53, MDM2, CREBBP, RB1, MDM2, CDKN2A/B, ATR, $M Y C, C H E K 2)$, Fanconi anemia pathway (FANCA, FANCD2, FANCI, SLX4, ATR), NOTCH signaling (NOTCH2 and NOTCH3), and/or PI3K-AKT signaling pathway (TSC1/2, AKT3, FGF19, STK11, MYC, FGFR1) as well as genes reported in endometrial cancers (POLE, ARIDIA, KRAS, FBXW7).

We reviewed p53 expression in more details. With immunohistochemistry, abnormal p53 expression was observed in nine tumors (three with diffuse overexpression and six with null expression). TP53 variants were further confirmed by our NGS in 4 tumors. Carleton et. al. reported abnormal (mutation type) staining in pattern in 19 of the 46 patients in their cohort [14]. Furthermore, our data revealed that MDM2 12q15 amplifications were exclusively present in p53 normal tumors. Mutual exclusivity between TP53 variant and MDM2 12q15 amplifications was previously observed in soft tissue sarcomas [36] and is suggestive of existence of alternative mechanisms to regulate the same cellular process. Interestingly, MDM2 12q15 amplifications are currently used as a diagnostic tool to identify suspected well-differentiated and de-differentiated liposarcomas. Therefore, $M D M 2$ amplifications might potentially serve as a valuable tool in the diagnosis of p53 normal gastric-type endocervical adenocarcinoma patients and also sheds light on the possibility of using MDM2 antagonists in treating this subset of tumors.

TP53-overexpressing tumors in our cohort also exhibited frequent $P O L E$ aberrations. This was an unexpected finding and for this reason we reviewed all cases with POLE gene aberrations to exclude the possibility of an endometrial primary being misclassified as gastric-type endocervical adenocarcinoma. All cases with POLE variant were located within cervix and showed classic histological features of gastric-type endocervical adenocarcinoma. Recent TCGA data has revealed that tumors harboring POLE variants can 
be targeted by immune checkpoint inhibitors. It will be informative to further explore the spectrum of POLE aberrations in a larger set of gastric-type endocervical adenocarcinoma tumors [37] and their response to immunotherapy. Lastly, $80 \%$ of p53-null tumors were found to contain aberrations in $C D K N 2 A / B$ genes. This is suggestive of aberrant cell cycle control in these types of tumors, and may serve as a bona fide rationale for targeting these tumors with cell cycle inhibitors. Our overall NGS results (POLE, ARIDIA, KRAS, FBXW7 variants) suggest that these tumors resemble endometrial cancers more closely than the usual type endocervical carcinomas in terms of molecular features $[38,39]$.

Gastric-type endocervical adenocarcinoma may be associated with genetic predisposition such as that seen in Peutz-Jeghers syndrome, known for association with hamartomatous gastrointestinal polyps and mucocutaneous pigmentation. Peutz-Jeghers syndrome is an autosomaldominant disorder that carries a mutation in the tumorsuppressor gene, STK11 (chromosome 19p13.3). There is an increased lifetime risk of ovarian, breast, gastric, pancreatic, and lung cancer in the affected individuals [40-42]. Approximately $14 \%$ of gastric-type endocervical adenocarcinoma tumors have been reported to be associated with Peutz-Jeghers syndrome [25, 27, 43]. LEGH lesions have also been found in some Peutz-Jeghers syndrome cases, and STK11 variants are found in some LEGH [24, 44]. Kim et al. highlighted the association of STK11 mutation with mucinous glandular lesions of pyloric differentiation in the female genital tract [45]. In addition, one case report has shown that gastric-type endocervical adenocarcinoma was identified in a patient with Lynch syndrome [46].

Mutation of STK11 gene has been implicated in a subset of LEGH, gastric-type endocervical adenocarcinoma and in Peutz-Jeghers syndrome $[12,47]$. Our findings showed that four cases were with STK11 null (frameshift/nonsense) variants, underlining the need for surveillance for Peutz-Jeghers syndrome-affected individuals [27]. In addition, our panel showed MSH6, MLH1, MSH2, and $P M S 2$ aberrations. Although there is a case report of gastrictype endocervical adenocarcinoma associated with mismatch repair genes and Lynch syndrome [12, 46], however, all of our cases showed intact expression of mismatch repair markers on immunohistochemical staining. As it has been well documented in the literature that there is a high degree of concordance between microsatellite instability (MSI) and immunohistochemical expression of mismatch repair markers, we did not pursue performing MSI on tumors.

HER2 amplification by FISH in gastric-type endocervical adenocarcinoma has not been investigated extensively so far [14].

The HER2 amplification in $20 \%$ of patients in our series is significant as it may have treatment implications. Patients with HER2 amplification died of disease, suggesting a possible impact of HER2 amplification on survival outcome; however, we acknowledge that this is a very small cohort and the results need to be further validated in a larger series.

In conclusion, our molecular results provide rare and valuable insight into genetic heterogeneity and aberrations implicated in gastric-type endocervical adenocarcinoma, which warrant further investigation for additional treatment and management options in larger preclinical studies and eventually clinical trials.

Funding support This work was supported by operating grants from Ontario Molecular Pathology Research Network-CPTGR-007.

\section{Compliance with ethical standards}

Conflict of interest The authors declare that they have no conflict of interest.

Publisher's note: Springer Nature remains neutral with regard to jurisdictional claims in published maps and institutional affiliations.

\section{References}

1. Pimenta JM, Galindo C, Jenkins D, Taylor SM. Estimate of the global burden of cervical adenocarcinoma and potential impact of prophylactic human papillomavirus vaccination. BMC Cancer. 2013;13:553.

2. Pirog EC, Lloveras B, Molijn A, Tous S, Guimera N, Alejo M, et al. HPV prevalence and genotypes in different histological subtypes of cervical adenocarcinoma, a worldwide analysis of 760 cases. Mod Pathol. 2014;27:1559-67.

3. Holl K, Nowakowski AM, Powell N, McCluggage WG, Pirog EC, Collas De Souza $S$, et al. Human papillomavirus prevalence and type-distribution in cervical glandular neoplasias: results from a European multinational epidemiological study. Int $\mathrm{J}$ Cancer. 2015;137:2858-68.

4. Quint KD, de Koning MN, Geraets DT, Quint WG, Pirog EC. Comprehensive analysis of Human Papillomavirus and Chlamydia trachomatis in in-situ and invasive cervical adenocarcinoma. Gynecol Oncol. 2009;114:390-4.

5. Mikami Y, Hata S, Fujiwara K, Imajo Y, Kohno I, Manabe T. Florid endocervical glandular hyperplasia with intestinal and pyloric gland metaplasia: worrisome benign mimic of "adenoma malignum". Gynecol Oncol. 1999;74:504-11.

6. Mikami Y, Hata S, Melamed J, Fujiwara K, Manabe T. Lobular endocervical glandular hyperplasia is a metaplastic process with a pyloric gland phenotype. Histopathology. 2001;39:364-72.

7. Mikami Y, Kiyokawa T, Hata S, Fujiwara K, Moriya T, Sasano H, et al. Gastrointestinal immunophenotype in adenocarcinomas of the uterine cervix and related glandular lesions: a possible link between lobular endocervical glandular hyperplasia/pyloric gland metaplasia and 'adenoma malignum'. Mod Pathol. 2004; 17:962-72.

8. Nucci MR, Clement PB, Young RH. Lobular endocervical glandular hyperplasia, not otherwise specified: a clinicopathologic analysis of thirteen cases of a distinctive pseudoneoplastic lesion and comparison with fourteen cases of adenoma malignum. Am J Surg Pathol. 1999;23:886-91. 
9. McCluggage WG. New developments in endocervical glandular lesions. Histopathology. 2013;62:138-60.

10. Ishii K, Hosaka N, Toki T, Momose M, Hidaka E, Tsuchiya S, et al. A new view of the so-called adenoma malignum of the uterine cervix. Virchows Arch. 1998;432:315-22.

11. Kurman RJ, International Agency for Research on Cancer, World Health Organization. WHO classification of tumours of female reproductive organs. 4th ed. Lyon: International Agency for Research on Cancer; 2014. 307 p.

12. Talia KL, McCluggage WG. The developing spectrum of gastrictype cervical glandular lesions. Pathology. 2018:50;122-33.

13. Kojima A, Mikami Y, Sudo T, Yamaguchi S, Kusanagi Y, Ito M, et al. Gastric morphology and immunophenotype predict poor outcome in mucinous adenocarcinoma of the uterine cervix. Am J Surg Pathol. 2007;31:664-72.

14. Carleton C, Hoang L, Sah S, Kiyokawa T, Karamurzin YS, Talia $\mathrm{KL}$, et al. A detailed immunohistochemical analysis of a large series of cervical and vaginal gastric-type adenocarcinomas. Am J Surg Pathol. 2016;40:636-44.

15. Mikami Y, McCluggage WG. Endocervical glandular lesions exhibiting gastric differentiation: an emerging spectrum of benign, premalignant, and malignant lesions. Adv Anat Pathol. 2013;20:227-37

16. Karamurzin YS, Kiyokawa T, Parkash V, Jotwani AR, Patel P, Pike MC, et al. Gastric-type endocervical adenocarcinoma: an aggressive tumor with unusual metastatic patterns and poor prognosis. Am J Surg Pathol. 2015;39:1449-57.

17. Bartley AN, Christ J, Fitzgibbons PL, Hamilton SR, Kakar S, Shah MA, et al. Template for reporting results of HER2 (ERBB2) biomarker testing of specimens from patients with adenocarcinoma of the stomach or esophagogastric junction. Arch Pathol Lab Med. 2015;139:618-20.

18. Wolff AC, Hammond ME, Hicks DG, Dowsett M, McShane LM, Allison KH, et al. Recommendations for human epidermal growth factor receptor 2 testing in breast cancer: American Society of Clinical Oncology/College of American Pathologists clinical practice guideline update. J Clin Oncol. 2013;31:3997-4013.

19. Kent WJ, Sugnet CW, Furey TS, Roskin KM, Pringle TH, Zahler AM, et al. The human genome browser at UCSC. Genome Res. 2002;12:996-1006.

20. Landrum MJ, Lee JM, Benson M, Brown GR, Chao C, Chitipiralla $\mathrm{S}$, et al. ClinVar: improving access to variant interpretations and supporting evidence. Nucleic acids Res. 2017;46: D1062-7.

21. Consortium GP. A global reference for human genetic variation. Nature. 2015;526:68-74.

22. Exome Variant Server, NHLBI GO Exome Sequencing Project (ESP), Seattle, WA (URL: http://evs.gs.washington.edu/EVS/).

23. Lek M, Karczewski KJ, Minikel EV, Samocha KE, Banks E, Fennell T, et al. Analysis of protein-coding genetic variation in 60,706 humans. Nature. 2016;536:285-91.

24. Matsubara A, Sekine S, Ogawa R, Yoshida M, Kasamatsu T, Tsuda $\mathrm{H}$, et al. Lobular endocervical glandular hyperplasia is a neoplastic entity with frequent activating GNAS mutations. Am J Surg Pathol. 2014;38:370-6.

25. Gilks CB, Young RH, Aguirre P, DeLellis RA, Scully RE. Adenoma malignum (minimal deviation adenocarcinoma) of the uterine cervix. A clinicopathological and immunohistochemical analysis of 26 cases. Am J Surg Pathol. 1989;13:717-29.

26. Kojima A, Shimada M, Mikami Y, Nagao S, Takeshima N, Sugiyama $\mathrm{T}$, et al. Chemoresistance of gastric-type mucinous carcinoma of the uterine cervix: a study of the Sankai Gynecology Study Group. Int J Gynecol Cancer. 2018;28:99-106.

27. Garg K, Karnezis AN, Rabban JT. Uncommon hereditary gynaecological tumour syndromes: pathological features in tumours that may predict risk for a germline mutation. Pathology. 2018;50:238-56.

28. Talia KL, Stewart CJR, Howitt BE, Nucci MR, McCluggage WG. HPV-negative gastric type adenocarcinoma in situ of the cervix: a spectrum of rare lesions exhibiting gastric and intestinal differentiation. Am J Surg Pathol. 2017;41:1023-33.

29. Wada T, Ohishi Y, Kaku T, Aman M, Imamura H, Yasutake N, et al. Endocervical adenocarcinoma with morphologic features of both usual and gastric types: clinicopathologic and immunohistochemical analyses and high-risk hpv detection by in situ hybridization. Am J Surg Pathol. 2017;41:696-705.

30. Ishii K, Katsuyama T, Ota H, Watanabe T, Matsuyama I, Tsuchiya S, et al. Cytologic and cytochemical features of adenoma malignum of the uterine cervix. Cancer. 1999;87:245-53.

31. Xu JY, Hashi A, Kondo T, Yuminamochi T, Nara M, Hashi K, et al. Absence of human papillomavirus infection in minimal deviation adenocarcinoma and lobular endocervical glandular hyperplasia. Int J Gynecol Pathol. 2005;24:296-302.

32. Kondo T, Hashi A, Murata SI, Fischer SE, Nara M, Nakazawa T, et al. Gastric mucin is expressed in a subset of endocervical tunnel clusters: type A tunnel clusters of gastric phenotype. Histopathology. 2007;50:843-50.

33. Kusanagi Y, Kojima A, Mikami Y, Kiyokawa T, Sudo T, Yamaguchi S, et al. Absence of high-risk human papillomavirus (HPV) detection in endocervical adenocarcinoma with gastric morphology and phenotype. Am J Pathol. 2010;177:2169-75.

34. McCluggage WG. Recent developments in non-HPV-related adenocarcinomas of the lower female genital tract and their precursors. Adv Anat Pathol. 2016;23:58-69.

35. Ohta Y, Suzuki T, Hamatani S, Shiokawa A, Kushima M, Ota H. Lobular endocervical glandular hyperplasia might become a precursor of adenocarcinoma with pyloric gland features. Pathol Res Pract. 2008;204:683-7.

36. Leach FS, Tokino T, Meltzer P, Burrell M, Oliner JD, Smith S, et al. p53 Mutation and MDM2 amplification in human soft tissue sarcomas. Cancer Res. 1993;53:2231-4.

37. Mehnert JM, Panda A, Zhong H, Hirshfield K, Damare S, Lane $\mathrm{K}$, et al. Immune activation and response to pembrolizumab in POLE-mutant endometrial cancer. J Clin Investig. 2016; 126:2334-40.

38. Cancer Genome Atlas Research Network. Integrated genomic characterization of endometrial carcinoma. Nature. 2013; 497:67-73.

39. Cancer Genome Atlas Research Network. Integrated genomic and molecular characterization of cervical cancer. Nature. 2017;543:378.

40. Beggs AD, Latchford AR, Vasen HF, Moslein G, Alonso A, Aretz $\mathrm{S}$, et al. Peutz-Jeghers syndrome: a systematic review and recommendations for management. Gut. 2010;59:975-86.

41. Aretz S, Stienen D, Uhlhaas S, Loff S, Back W, Pagenstecher C, et al. High proportion of large genomic STK11 deletions in PeutzJeghers syndrome. Hum Mutat. 2005;26:513-9.

42. Giardiello FM, Brensinger JD, Tersmette AC, Goodman SN, Petersen GM, Booker SV, et al. Very high risk of cancer in familial Peutz-Jeghers syndrome. Gastroenterology. 2000;119:1447-53.

43. Chen KT. Female genital tract tumors in Peutz-Jeghers syndrome. Hum Pathol. 1986;17:858-61.

44. Hirasawa A, Akahane T, Tsuruta T, Kobayashi Y, Masuda K, Banno $\mathrm{K}$, et al. Lobular endocervical glandular hyperplasia and peritoneal pigmentation associated with Peutz-Jeghers syndrome due to a germline mutation of STK11. Ann Oncol. 2012;23:2990-2.

45. Kim EN, Kim GH, Kim J, Park IA, Shin JH, Chai Y, et al. A pyloric gland-phenotype ovarian mucinous tumor resembling 
lobular endocervical glandular hyperplasia in a patient with PeutzJeghers syndrome. J Pathol Transl Med. 2017;51:159-64.

46. Moat M, O’Donnell RL, McCluggage WG, Ralte A, Edmondson

RJ. Gastric-type adenocarcinoma of the cervix in a patient with Lynch syndrome: a case report. Gynecol Oncol Rep. 2014;10:41-3.
47. Kuragaki C, Enomoto T, Ueno Y, Sun H, Fujita M, Nakashima R, et al. Mutations in the STK11 gene characterize minimal deviation adenocarcinoma of the uterine cervix. Lab Invest. 2003;83:35-45. 